NISTIR 7967

\title{
Compensating Operators and Stable Backward in Time Marching in Nonlinear Parabolic Equations
}

Alfred S. Carasso

http://dx.doi.org/10.6028/NIST.IR.7967 
NISTIR 7967

\title{
Compensating Operators and Stable Backward in Time Marching in Nonlinear Parabolic Equations
}

\author{
Alfred S. Carasso \\ Applied and Computational Mathematics Division \\ Information Technology Laboratory
}

http://dx.doi.org/10.6028/NIST.IR.7967

November 2013

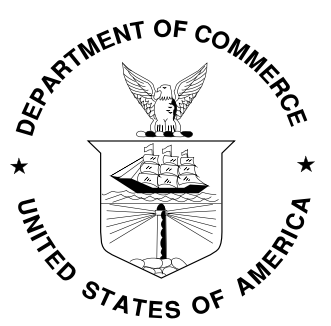

U.S. Department of Commerce

Penny Pritzker, Secretary

National Institute of Standards and Technology Patrick D. Gallagher, Under Secretary of Commerce for Standards and Technology and Director 


\title{
COMPENSATING OPERATORS AND STABLE BACKWARD IN TIME MARCHING IN NONLINEAR PARABOLIC EQUATIONS.
}

\author{
ALFRED S. CARASSO*
}

\begin{abstract}
Step by step time-marching schemes are fundamental tools in the numerical exploration of well-posed nonlinear evolutionary partial differential equations. However, when the initial value problem is ill-posed, such stepwise numerical schemes are necessarily unconditionally unstable and result in explosive noise amplification. This paper outlines a novel stabilized time-marching procedure for computing nonlinear parabolic equations on 2D rectangular regions, backward in time. Very little is known either analytically, or computationally, about this class of exponentially ill-posed problems. To quench the instability, the procedure uses easily synthesized FFT-based compensating operators at every time step. A fictitious nonlinear image deblurring problem is used to evaluate the effectiveness of this computational approach. The method is compared with a previously introduced, global in time nonlinear Van Cittert iterative procedure. The latter is significantly more time consuming, and impractical on large problems.
\end{abstract}

Key words. nonlinear backward parabolic equations; ill-posed initial value problem; backward time-marching scheme; FFT compensating operators; non-integer power Laplacian; nonlinear Van Cittert method; nonlinear image deblurring.

AMS subject classifications. 35K55, 35R25, 65M06, 65M30, 68U10.

1. Introduction. Reconstructing the past behavior of a physical system from knowledge of its current state, is of interest in numerous scientific contexts. However, when the physical process involves diffusion, as is the case in groundwater contamination studies for example, the resulting backward parabolic reconstructions are exponentially ill-posed and present serious computational issues [1], [2], [3]. Similar exponential ill-posedness characterizes elliptic downward continuation of satellite measurements of gravity and geomagnetism [4], [5], [6]. In recent years, significant progress has been made in successfully solving linear time-reversed parabolic equations, leading to useful reconstructions of valuable scientific data in astronomy, medicine, and other areas of application [7]. However, little is known about backward continuation in multidimensional nonlinear parabolic equations, either analytically or computationally.

This paper outlines a novel time-marching procedure for computing time-reversed nonlinear parabolic equations on $2 \mathrm{D}$ rectangular regions. As is well-known, stepwise marching schemes consistent with ill-posed initial value problems are necessarily computationally unstable. Here, easily synthesized compensating operators, based on FFT algorithms, are applied at every time step to quench the instability. A fictitious image deblurring problem is used to evaluate the effectiveness of this computational approach, as well as to probe the behavior of various types of nonlinearities. Such $c a$ nary in the coalmine synthetically blurred images are useful indicators of the type and quality of information that might be recoverable, when the backward computation is applied to real data in engineering contexts unrelated to imaging. Further exploration and refinement of this time-marching methodology will be developed in subsequent reports. Similar ideas may be applicable in ill-posed initial value problems associated with other types of partial differential equations.

Recently [8], a global in time iterative procedure was successfully applied to a class of $2 \mathrm{D}$ nonlinear backward parabolic equations. This method is an adaptation to the

*Applied and Computational Mathematics Division, National Institute of Standards and Technology, Gaithersburg, MD 20899. (alfred.carasso@nist.gov). 
nonlinear case of the Van Cittert iteration [9], originally used in spectroscopy in the 1930's to solve 1D linear convolution integral equations, with explicitly known kernels having positive Fourier transforms. It is remarkable that the Van Cittert method works as well as it does on the limited, but significant class of nonlinear problems considered in [8]. However, the nonlinear Van Cittert process becomes prohibitively time consuming on large problems, such as $512 \times 512$ or $1024 \times 1024$ pixel images. As will be shown below, when backward continuation is feasible, the new stepwise marching scheme can produce results with almost the same visual quality as in the Van Cittert iteration, but almost 80 times faster.

2. Limited backward recovery in nonlinear parabolic problems. Most of the useful computational results in backward in time parabolic continuation have generally been obtained in the canonical case of linear, autonomous, selfadjoint problems. As shown in [3], [8], nonlinear parabolic equations deviating too strongly from this canonical model can present additional challenges. The reason for this marked difference in behavior can be traced back to the Hölder-continuity stability inequalities that underlie backward reconstruction from noisy data.

Let $\Omega$ be a bounded domain in $R^{n}$ with smooth boundary $\partial \Omega$. Let $L$ be a linear or nonlinear elliptic operator in $\Omega$, acting on smoothly differentiable functions satisfying homogeneous Dirichlet or Neumannn conditions on $\partial \Omega$. Let $L$ be such that the forward initial value problem $w_{t}=L w, t>0, w(0)=g(x)$, is well-posed in $L^{2}(\Omega)$. Let $w^{1}(x, t)$ and $w^{2}(x, t)$ be any two solutions, and let $F(t)=\left\|w^{1}(., t)-w^{2}(., t)\right\|_{2}^{2}$, for $0 \leq t \leq T$.

Using logarithmic convexity techniques [10], the folowing sharp inequality can be established for a wide class of parabolic equations $w_{t}=L w$,

$$
F(t) \leq\{F(0)\}^{1-\mu(t)}\{F(T)\}^{\mu(t)}, \quad 0 \leq t \leq T .
$$

Here, the Hölder exponent $\mu(t)$ satisfies $0 \leq \mu(t) \leq 1$, with $\mu(t)>0, t>0, \mu(T)=$ $1, \mu(0)=0$, and $\mu(t) \downarrow 0$ monotonically as $t \downarrow 0$.

Given noisy data $f(x) \in L^{2}(\Omega)$ at time $T$, satisfying $\|w(., T)-f\|_{2} \leq \delta$ for small $\delta>0$, we restrict attention to backward continuations $w(x, t)$ satisfying a prescribed bound at $t=0$, i.e., $\|w(., 0)\|_{2} \leq M$. Eq. (2.1) then implies the following stability inequality for the difference of any two possible continuations $w^{1}(x, t), w^{2}(x, t)$, from the data $f(x)$ at $t=T$,

$$
\left\|w^{1}(., t)-w^{2}(., t)\right\|_{2} \leq 2 M^{1-\mu(t)} \delta^{\mu(t)}, \quad 0 \leq t \leq T .
$$

In the linear autonomous selfadjoint case, the Hölder exponent $\mu(t)$ in Eq. (2.2) decays linearly to zero, as $t \downarrow 0$. In the nonlinear case, rapid exponential decay of $\mu(t)$ to zero is possible [3], [10]. Therefore, a level of accuracy $\delta$ at time $T$ that can produce useful backward reconstruction in an autonomous selfadjoint equation, may be insufficient in a nonlinear equation. As a rule, high quality nonlinear reconstructions from imprecise data are not easily achieved. Examples of physically plausible, smooth, positive, yet false nonlinear reconstructions are given in [3]. In applications involving real data with limited but unknown precision, detailed prior information about the true solution may be critical in evaluating the merits of a candidate reconstruction.

3. Two nonlinear parabolic examples $w_{t}=L w$. The computational experiments discussed below involve four images and two instructive parabolic equations. However, numerous other equations can be studied, exhibiting a wide variety of unexpected phenomena. Let $\Omega$ be the unit square $0<x, y<1$ in the $(x, y)$ plane. With 
fixed $T>0$, and homogeneous Neumann boundary conditions on $\partial \Omega$, the following initial value problem will be studied,

$$
\begin{aligned}
& w_{t}=\kappa s(w) \nabla \cdot\{q(x, y, t) \nabla w\}+c w w_{x},+d\left(w \cos ^{2} w\right) w_{y}, \quad \Omega \times(0, T), \\
& w(x, y, 0)=g(x, y) .
\end{aligned}
$$

Here $\kappa=8.5 \times 10^{-4}, c=1.25, d=0.6$, and

$$
\begin{aligned}
& s(w)=\exp (0.025 w), \\
& q(x, y, t)=\exp (10 t)\left(1+5 e^{2 y} \sin \pi x\right) \geq 1, \quad \Omega \times(0, T) .
\end{aligned}
$$

An equation with different nonlinearities will also be considered. This is

$$
\begin{aligned}
& w_{t}=\gamma r(w) \nabla \cdot\{q(x, y, t) \nabla w\}+a \sqrt{|w|} w_{x},+b\left(w \cos ^{2} w\right) w_{y}, \quad \Omega \times(0, T), \\
& w(x, y, 0)=g(x, y),
\end{aligned}
$$

where $\gamma=0.04, a=b=0.5, r(w)=\exp \left\{\cos ^{2}(w)\right\}$, and $q(x, y, t)$ is as previously defined in Eq. (3.2).

Each of Eqs.(3.1), (3.3), is well posed in $L^{2}(\Omega)$. Accordingly, given any initial value $w(x, y, 0)=g(x, y) \in L^{2}(\Omega)$, a unique solution $w(x, y, T)$ exists at time $T$, and the solution operator $\Lambda^{T}$, where

$$
\Lambda^{T} w(x, y, 0)=w(x, y, T),
$$

is well-defined on $L^{2}(\Omega)$. The nonlinear operator $\Lambda^{T}$ is not known explicitly. Rather, $\Lambda^{T} w(x, y, 0)$ must be found by solving the appropriate initial value problem Eq.(3.1), or Eq.(3.3), and obtaining the corresponding solution at time $T$. Note that $w(x, y, T)$ necessarily belongs to a very restricted class of smooth functions.

4. Explicit scheme for computing $w_{t}=L w$ forward in time. Step by step time marching explicit finite difference schemes are simple, convenient, and effective tools for solving nonlinear evolutionary partial differential equations, as they allow lagging the nonlinearities at the previous time step. Despite the necessary stability restriction on the time step $\Delta t$, such schemes enable a user to quickly gain a preliminary indication of the salient characteristics of the unknown solution. Higher precison numerics, along with unconditionally stable implicit schemes, may be applied at a subsequent stage. Here, using explicit time differencing and centered space differencing in the nonlinear initial value problems in Eqs.(3.1) and (3.3), leads to modest $O\left(\Delta t+(\Delta x)^{2}+(\Delta y)^{2}\right)$ accuracy. However, the explicit stability condition on $\Delta t$ improves that accuracy to $O\left((\Delta x)^{2}+(\Delta y)^{2}\right)$. This paper deals with 8 bit gray scale $I M \times I M$ pixel images, with pixel values ranging between 0 and 255 . With $\Delta x=\Delta y=1 / I M, \Delta t=T / N T$, the following difference approximation is used to march the discrete mesh function $W^{n} \equiv W(j \Delta x, k \Delta y, n \Delta t)$ in Eq. (3.1), $N T$ time steps $\Delta t$ forward in time, up to time $T=N T \Delta t$,

$$
\begin{aligned}
W^{n+1} & =W^{n}+\Delta t \kappa S\left(W^{n}\right) \nabla \cdot\left\{Q^{n} \nabla W^{n}\right\}+c W^{n} W_{x}^{n} \\
& +d\left(W^{n} \cos ^{2} W^{n}\right) W_{y}^{n}, \quad n=0, N T-1, \\
W^{0} & =g(x, y) .
\end{aligned}
$$


Similar differencing is applied to Eq. (3.3). Homogeneous Neumann conditions are applied on the boundary of the unit square. In this notation, $W_{x}, W_{y}, \nabla \cdot\{Q \nabla W\}$, represent centered finite difference approximations in the space variables, $W^{0}$ denotes the original sharp image $g(x, y)$, while $W^{N T}$ is the nonlinearly blurred image $f(x, y)$, using either Eq.(3.1) or Eq.(3.3). Analogously to the analytic problem in Eq. (3.4), we define the discrete nonlinear parabolic blurring operator $\Lambda_{d}^{T}$ by

$$
\Lambda_{d}^{T} W^{0}=W^{N T} \quad \Longleftrightarrow \quad \Lambda_{d}^{T} g(x, y)=f(x, y) .
$$

This nonlinear operator is defined on any 8 bit gray scale $I M \times I M$ pixel image $g(x, y)=W^{0}$. Applying $\Lambda_{d}^{T}$ to that image simply means applying the above explicit scheme for $N T$ time steps to $W^{0}$, and acquiring the resulting array $f(x, y)=W^{N T}$. We stress that the blurred image $f(x, y)$ so obtained is only an approximation to the true solution $w(x, y, T)$ in Eq.(3.1) or Eq.(3.3).

5. Backward continuation in $w_{t}=L w$ and the Van Cittert iteration. In its original formulation, given the data $f(x)$ and the explicitly known 1D linear convolution integral operator $P$ with Fourier transform $\hat{P}(\xi)>0$, the Van Cittert method solves $P g=f$ for the unknown $g(x)$ by means of the iterative procedure

$$
h^{m+1}(x)=h^{m}(x)+\lambda\left\{f(x)-P\left[h^{m}(x)\right]\right\}, \quad m \geq 1 .
$$

Here, $\lambda>0$ is a fixed relaxation parameter chosen so that $1-\lambda \hat{P}(\xi)>0, h^{1}(x)=$ $\lambda f(x)$, and the expectation is that $h^{m} \rightarrow g$. Indeed, taking Fourier Transforms in Eq. (5.1), we find

$$
\hat{h}^{m+1}(\xi)=\left(\sum_{j=0}^{m}\{1-\lambda \hat{P}(\xi)\}^{j}\right) \lambda \hat{f}(\xi), \quad m \geq 1 .
$$

Since, $|1-\lambda \hat{P}(\xi)|<1$, one has $\sum_{j=0}^{\infty}\{(1-\lambda \hat{P}(\xi)\})^{j}=\{\lambda \hat{P}(\xi)\}^{-1}$. Hence, $\hat{h}^{m}(\xi)$ 'converges' to $\hat{g}(\xi)$ as $m \rightarrow \infty$, and $h^{m}(x) \rightarrow g(x)$. In reality, noise in the data $f(x)$, together with rapid decay of $\hat{P}(\xi)$ at infinity in commonly occurring Gaussian-like convolution operators $P$, lead to error amplification at high frequencies which prevents convergence. The advantage of the Van Cittert iteration is that it provides a gradual deconvolution process whereby low and moderately high frequency information can be recovered in the first few iterations, while many more iterations are necessary to obtain the remaining high frequency information. However, since such high frequency information is quite likely contaminated by amplified data noise, it is wise to monitor the iterative process and be able to terminate it before amplified noise begins to degrade the reconstruction. In spectroscopy and image processing applications [11], [12], the Van Cittert method is generally found to produce useful results after finitely many iterations, even though it may not converge.

We now contemplate applying the Van Cittert iteration in the present parabolic context to recover $w(x, y, 0)=g(x, y)$ in Eqs.(3.1) and (3.3), given approximate values $f(x, y)$ for the true solution $w(x, y, T)$ at time $T$. This amounts to inverting the discrete nonlinear operator $\Lambda_{d}^{T}$ in Eq. (4.2) by solving for the unknown $g(x, y)$ using the iterative procedure

$$
h^{m+1}(x, y)=h^{m}(x, y)+\lambda\left\{f(x, y)-\Lambda_{d}^{T} h^{m}(x, y)\right\}, \quad m \geq 1,
$$

with some fixed $\lambda$ such that $0<\lambda<1$, and $h^{1}(x, y)=\lambda f(x, y)$. While we may hope that $h^{m}(x, y) \rightarrow g(x, y)$, there are considerable theoretical and computational 
gaps between the iterative process in Eq. (5.1) and the one in Eq. (5.3). Unlike the fixed, explicitly known, linear convolution operator $P$ in Eq. (5.1), $\Lambda_{d}^{T}$ in Eq. (5.3) is unknown, and a new nonlinear initial value must be solved numerically to obtain $\Lambda_{d}^{T} h^{m}(x, y)$ for each $m$. In addition, the Neumann series expansion in Eq. (5.2) which underlies 'convergence' in Eq. (5.1), is not applicable in Eq. (5.3). Thus, the iterative process in Eq. (5.3) is liable to be computationally intensive, yet with little or no theoretical expectation of success. Nevertheless, remarkably, the Van Cittert iteration is found to be a valuable tool in a wide variety of $2 \mathrm{D}$ nonlinear backward parabolic equations. In many cases, this procedure generates iterates $h^{m}(x, y)$ such that the $L^{\infty}$ norm of the residual, $f-\Lambda_{d}^{T} h^{m} \infty$, decays quasi-monotonically to a reasonably small value after a finite number $N$ of iterations, and $h^{N}(x, y)$ is found to be a useful approximation to $w(x, y, 0)$.

6. FFT compensating operator $B$ on rectangular regions. Define the $2 \mathrm{D}$ Fourier transform of an $L^{1}\left(R^{2}\right)$ function $h(x, y)$ by

$$
\hat{h}(\xi, \eta) \equiv \int_{R^{2}} h(x, y) e^{-2 \pi i(\xi x+\eta y)} d x d y,
$$

For fixed $p>1$, and small $\epsilon>0$, consider the well-posed diffusion initial value problem in $R^{2}$ involving possibly non-integer powers of the negative Laplacian

$$
w_{t}=-\epsilon(-\Delta)^{p} w, \quad t>0, \quad w(x, y, 0)=h(x, y)
$$

This has the unique Fourier space solution

$$
\hat{w}(\xi, \eta, t)=\exp \left\{-\epsilon t\left[(2 \pi \xi)^{2}+(2 \pi \eta)^{2}\right]^{p}\right\} \hat{h}(\xi, \eta), \quad t>0,
$$

from which $w(x, y, t)$ can be found by inverse Fourier transformation

(6.4) $w(x, y, t)=\int_{R^{2}} \exp \{2 \pi i(\xi x+\eta y)\} \exp \left\{-\epsilon t\left[(2 \pi \xi)^{2}+(2 \pi \eta)^{2}\right]^{p}\right\} \hat{h}(\xi, \eta) d \xi d \eta$.

Now, let $h(x, y)$ be an 8-bit $I M \times I M$ pixel image. Using direct and inverse FFT algorithms, we can mimick the operations in Eqs. (6.3), (6.4), and apply the diffusion equation Eq.(6.2) to the image $h(x, y)$, to produce the smoothed image $w(x, y, t)$. For fixed $t>0$, such smoothing will depend strongly on the chosen values of $\epsilon$ and $p$ in Eqs. (6.3), (6.4).

Let $\Delta t$ be the fixed time step in a step by step finite difference marching computation involving an $I M \times I M$ image. Let $h(x, y)$ be the resulting image at some discrete time $t_{k}=k \Delta t$ in the evolving computation. Using FFT algorithms, for given $\epsilon, p$, we may apply the compensating linear operator $B(\epsilon, p, \Delta t)$ to $h(x, y)$, where $B h$ is defined by

$$
B(\epsilon, p, \Delta t) h(x, y)=w(x, y, \Delta t),
$$

with $w(x, y, t)$ as in in Eq. (6.4).

Compensating operators in non rectangular regions, based on $(-\Delta)^{p}$, are discussed in Section 9 below.

7. Explicit scheme for computing $w_{t}=L w$ backward in time. Given the blurred image $f(x, y)=\Lambda_{d}^{T} g(x, y)$ in Eq. (4.2), we can attempt to recover the original sharp image $g(x, y)$ by marching backward from $T=N T \Delta t$ in the explicit 
finite difference scheme in Eq. (4.1). This becomes possible provided we apply a properly chosen FFT compensating operator $B$ to the computed image $V$ at each time step, to control the ill-posedness. With pre-assigned $\epsilon, p$, the proposed explicit backward in time scheme corresponding to Eq. (4.1) is then the following

$$
\begin{aligned}
V & =W^{m}-\Delta t \kappa S\left(W^{m}\right) \nabla \cdot\left\{Q^{N T-m} \nabla W^{m}\right\}+c W^{m} W_{x}^{m} \\
& +d\left(W^{m} \cos ^{2} W^{m}\right) W_{y}^{m}, \quad m=0, N T-1, \\
W^{m+1} & =B V, \quad m=0, N T-1 . \\
W^{0} & =f(x, y) .
\end{aligned}
$$

Here, $W^{0}$ is the given blurred image at time $T=N T \Delta t$, and the scheme marches backward $N T$ time steps to produce the sharp image $W^{N T}$ at $t=0$. With obvious modifications, this can also be applied in backward in time continuation in Eq.(3.3). As may be expected, the choices of $p$ and $\epsilon$ are critical in obtaining good results, and several interactive trials may be necessary. However, there are infinitely many useful pairs $(\epsilon, p)$, and such a pair can usually be located fairly quickly.

A different type of marching scheme for a class of ill-posed initial value problems is discussed in [13].

7.1. Relation to the quasi-reversibility method. While close in spirit to the quasi-reversibilty (QR) method [14], the compensated scheme in Eq. (7.1) differs from QR in essential ways that render it more versatile, and more easily applicable to nonlinear problems. The QR method deals with various ill-posed problems in partial differential equations, including spatial continuation in elliptic equations, and boundary control and other problems in parabolic and non-parabolic evolution equations. Application of QR to backward in time parabolic equations is developed in Chapter 1 of [14]. There, linear equations

$$
w_{t}+A(t) w=0
$$

are considered, with $A(t)$ a linear elliptic partial differential operator of order $2 m$ in the space variables, with coefficients depending smoothly on space and time. The differential operator $A(t)$ need not be selfadjoint, but is such that the forward in time problem is well-posed. Backward continuation from given noisy data at time $T>0$ is accomplished by numerically marching back from $t=T$ in the modified equation

$$
w_{t}+A(t) w-\epsilon A^{*}(t) A(t) w=0
$$

with suitably preselected small $\epsilon>0$. For the simple heat equation $w_{t}=\Delta w$, the modified equation is $w_{t}=\Delta w+\epsilon \Delta^{2} w$. For that simple case, the authors also contemplate the use of alternative modified equations, such as $w_{t}=\Delta w+\epsilon(-\Delta)^{m} w$ with preselected positive integer $m$. Illustrative 1D linear computational examples, using Crank-Nicolson time differencing, are also given in [14, Chapter 1]. However, nonlinear backward problems are not considered. Indeed, the formulation in Eq. (7.3) involving the adjoint $A^{*}(t)$, is not applicable to nonlinear problems in an obvious way. In the case of the nonlinear second order parabolic equations (3.1), (3.3), a modified equation involving some appropriate nonlinear generalization of $A^{*}(t) A(t) w$, would result in a fairly complex fourth order nonlinear equation, and present a challenging computational task.

In the QR approach, a particular modified equation is selected and translated into a specific computational code, using various trial values of $\epsilon>0$. Exploring 
other possible modified equations within the same code would not be practical, and is not typically contemplated. Also, only positive integer powers of various partial differential operators enter the modified equations. A significant practical advantage of the compensated scheme in Eq. (7.1), or its counterpart in more general domains described in Section 9 below, is the ability to simultaneously explore non integer positive values of $p$, as well as positive values of $\epsilon$, within the same computational code. Interestingly, in the examples below involving the nonlinear second order parabolic equations (3.1), (3.3), relatively simple compensating operators $B$ based on $(-\Delta)^{p}$ with $2<p<3$, were found fully capable of controlling instability in Eq. (7.1).

8. Some examples. Figure 8.1 shows four sharp test images. These images are used to probe the behavior of the blurring nonlinear partial differential equations $w_{t}=L w$ in Section 3, as well as to evaluate the merits of the proposed backward in time continuation algorithms. Each of these images features areas of zero intensity near the edges. Such zero padding is important in applications involving FFT algorithms. All computations described below were carried out on an AMD Athlon 64 X2 $4880+$, running Centos 532 bit. $^{1}$ The computation times mentioned are intended to illustrate the possible advantages, or disadvantages, of various procedures.

Failure of nonlinear reconstruction. We begin by observing that useful backward reconstruction may not be feasible in the absence of sufficient precision $\delta$ in the given data at time $T$. Figure 8.2 is particularly instructive in the context of continuation backward in time in nonlinear parabolic equations. Each of the three $256 \times 256$ degraded images on the left hand side, was obtained by blurring the corresponding sharp image in Figure 8.1, using the nonlinear initial value problem Eqs.(3.1), (3.2). This was done using the explicit scheme in Eq.(4.1), with $\Delta x=\Delta y=1 / 256, \Delta t=3.0 \times 10^{-7}$, and marching the solution 400 steps $\Delta t$ forward, up to the final time $T=1.2 \times 10^{-4}$. These degraded images do not appear blurred beyond remediation. Indeed, in linear image deconvolution experiments, similarly blurred imagery can often be significantly improved. Here, useful recovery using the Van Cittert method was not possible. Indeed, the original blurred images on the left are more informative than the proposed reconstructions! This example illustrates the remarks in Section 2 regarding the significance of exponentially decaying Hölder exponents $\mu(t)$ in the backward stabilty estimate Eq. (2.2). Evidently, despite the small value of $\Delta t$ in Eq.(4.1), the blurred image $W^{N T}=f(x, y)$ is not a sufficiently close approximation to the true solution $w(x, y, T)$ in Eqs.(3.1), (3.2). Clearly, that nonlinear initial value problem is a difficult test case. All remaining examples in this paper will involve blurring using Eq.(3.3), an equation with milder nonlinearities.

US Air Force resolution chart. Figure 8.3 involves a $512 \times 512$ image, and is designed to show the possible merits of using the compensated backward in time explicit scheme in Eq. (7.1), versus the more orthodox nonlinear Van Cittert method in Eq. (5.3). The scheme in Eq. (4.1) was applied to Eq. (3.3), with $\Delta x=\Delta y=1 / 512, \Delta t=1.5 \times 10^{-7}$, and 600 time steps, to yield the blurred image at the final time $T=9.0 \times 10^{-5}$. That blurring computation was accomplished in 3 minutes. As seen in the leftmost image in Figure 8.3, due to the pronounced anisotropic character of $q(x, y, t)$ in Eq. (3.3), the vertical sequence of numbers near

\footnotetext{
${ }^{1}$ Mention of commmercial products or services in this report does not imply NIST appproval or endorsement of these products or services, nor does it imply that such products or services are necessarily the best available for the intended purpose.
} 
the right edge has almost been erased. However, after 100 Van Cittert iterations in the rightmost image in Figure 8.3, that vertical sequence is partially restored, along with noticeable improvements in other parts of the image. As may be expected, that Van Cittert reconstruction required almost 5 hours, as it necessitated 100 sequential nonlinear blurring computations, each lasting 3 minutes.

Using $\epsilon=3.5 \times 10^{-4}, p=2.5$, in the compensated backward explicit scheme in Eq. (7.1), produced the middle image in Figure 8.3. The vertical sequence of numbers near the right edge appears better defined in the middle image than in the rightmost Van Cittert image. However, certain other parts of the image are marginally better recovered in the Van Cittert restoration. The above choice of $(\epsilon, p)$ was arrived at after several interactive trials, each lasting 4 minutes. Clearly, on a parallel computer, several such pairs $(\epsilon, p)$ can be explored simultaneously. No parameter choice other than $\lambda$ in Eq. (5.3) is necessary in the Van Cittert method.

Sagittal Brain MRI image. The $256 \times 256$ MRI Brain image in Figure 8.4 was blurred by applying the scheme in Eq.(4.1) to Eq. (3.3), with $\Delta x=\Delta y=$ $1 / 256, \Delta t=3.0 \times 10^{-7}$, and capturing the resulting image after 400 time steps at the final time $T=1.2 \times 10^{-4}$. That computation required 20 seconds. As in Figure 8.3, there is substantially more blurring near the right edge of the blurred image. A competent restoration results after 100 Van Cittert iterations and 2000 seconds, as shown in the rightmost image in Figure 8.4. However, after a few interactive trials, each requiring 25 seconds, the compensated backward explicit scheme in Eq. (7.1) with $\epsilon=2.25 \times 10^{-5}, p=2.875$, produced the almost as good reconstruction shown in the middle image in Figure 8.4.

Ben Franklin image. Identical mesh parameters were used to produce the $256 \times 256$ blurred Ben Franklin image in Figure 8.5. Recovery of the right half of that image is again challenging. Quite useful reconstruction was achieved after 100 Van Cittert iterations and 2000 seconds, as shown in the rightmost image in Figure 8.5. However, after a few 25 second trials, reasonably good recovery was achieved with the compensated backward explicit scheme in Eq. (7.1), using $\epsilon=4.0 \times 10^{-5}, p=2.775$. As in the preceding MRI Brain image, the Van Cittert image is again somewhat sharper.

Satellite image. Behavior in the $256 \times 256$ Satellite image differs from the preceding two cases, although identical mesh parameters were applied to generate the blurred image in Figure 8.6, using Eq. (3.3). That blurred image appears more degraded than the blurred Satellite image in Figure 8.2, where a more severe nonlinear process was used. In contrast to the case in Figure 8.2, very useful reconstruction is achieved after 100 Van Cittert iterations and 2000 seconds, as shown in the rightmost image in Figure 8.6. Here, interestingly, after a few 25 second interactive trials, equally good results can be achieved using the compensated backward explicit scheme in Eq. (7.1) with $\epsilon=6.5 \times 10^{-4}, p=2.35$. Behavior in this example is similar to that found in the previously discussed $512 \times 512$ US Air Force resolution chart.

9. Compensating operators $B$ in general domains. One may consider backward in time continuation in nonlinear second order parabolic problems on general domains $\Omega$ in $R^{2}$, with homogeneous Dirichlet or Neumann boundary conditions. Stabilizing backward in time marching computations by means of a compensating operator $B$ can be accomplished as follows. For the selfadjoint spatial differential 


\section{SHARP TEST IMAGES IN NONLINEAR DEBLURRING EXPERIMENTS}

Resolution chart

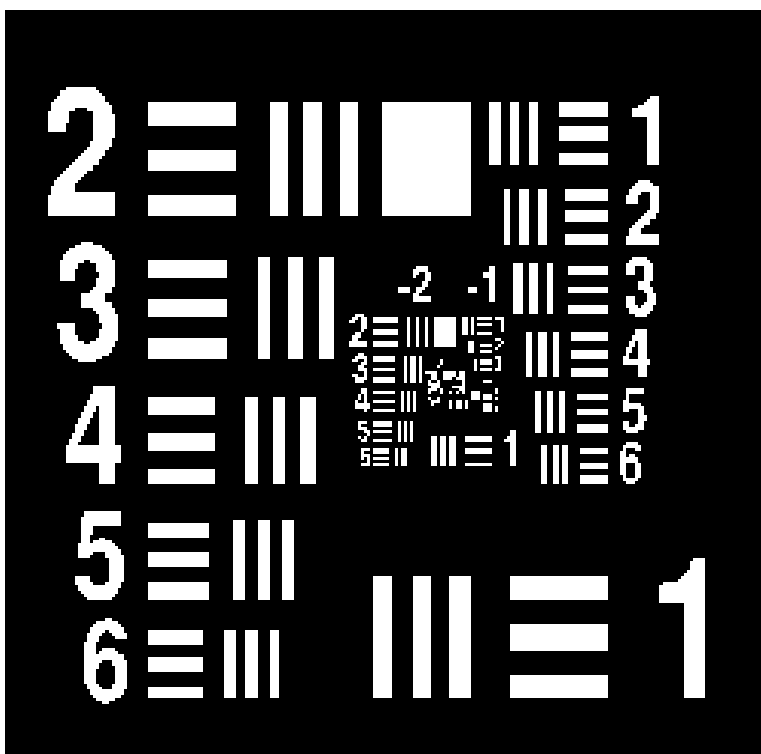

Ben Franklin

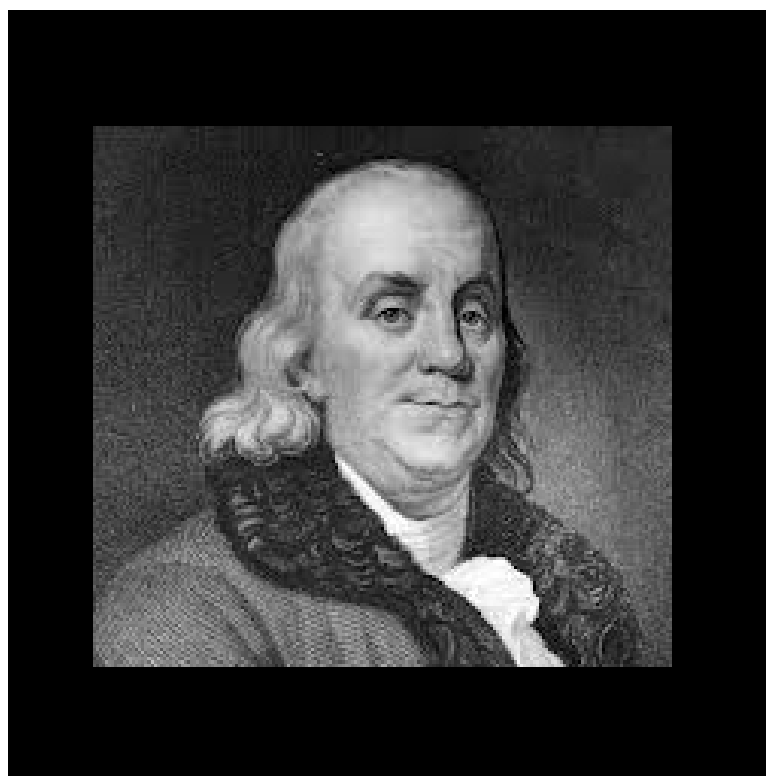

Brain MRI

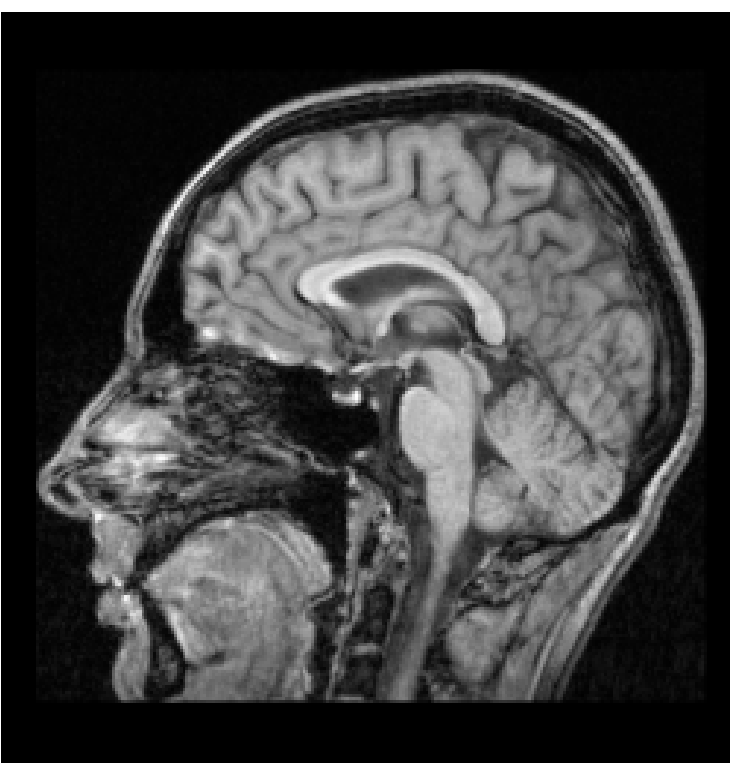

Satellite

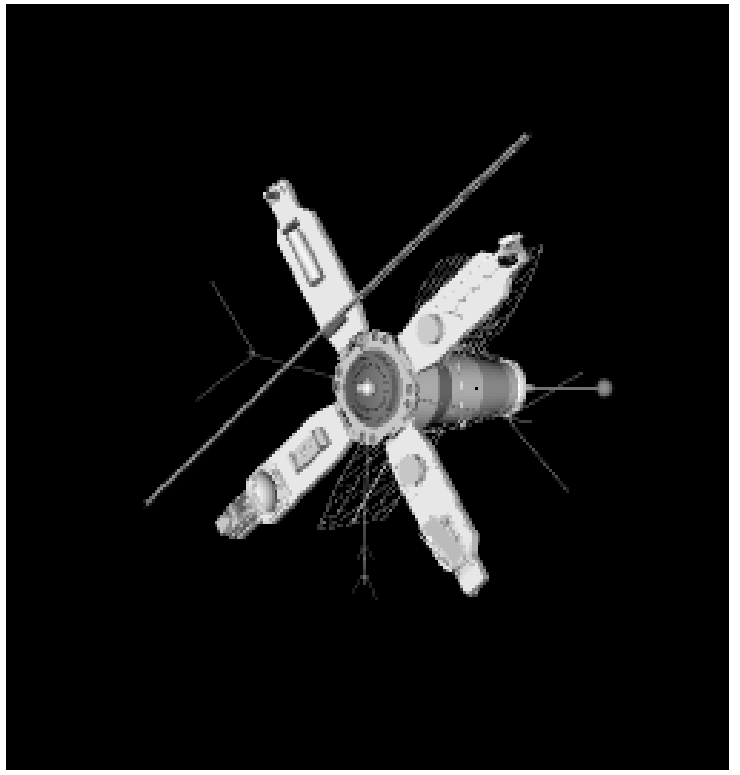

FIG. 8.1. A collection of test images is useful in exploring the feasibility of backward in time continuation in nonlinear parabolic equations, even when the intended application is unrelated to imaging. 
FAILED BACKWARD IN TIME RECONSTRUCTIONS IN NONLINEAR PARABOLIC EQUATION (3.1-3.2).

BLURRED IMAGE USING EQ. (3.1)

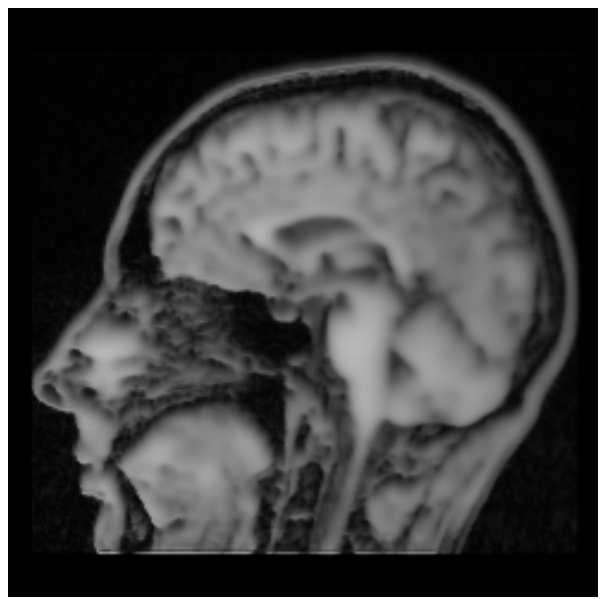

BLURRED IMAGE USING EQ. (3.1)

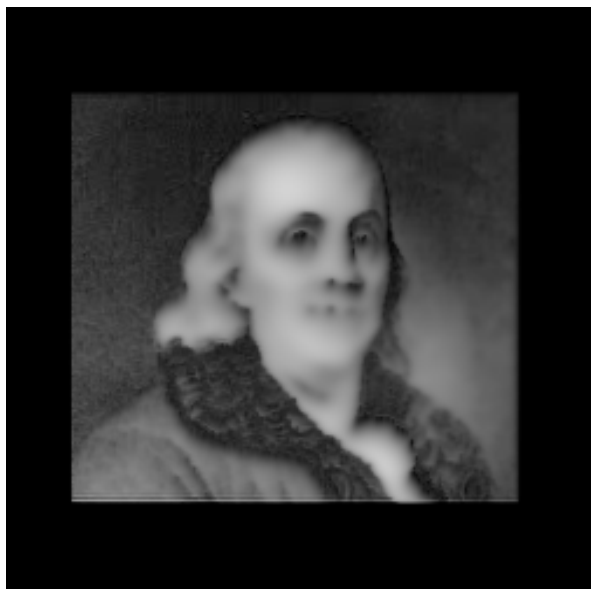

BLURRED IMAGE USING EQ. (3.1)

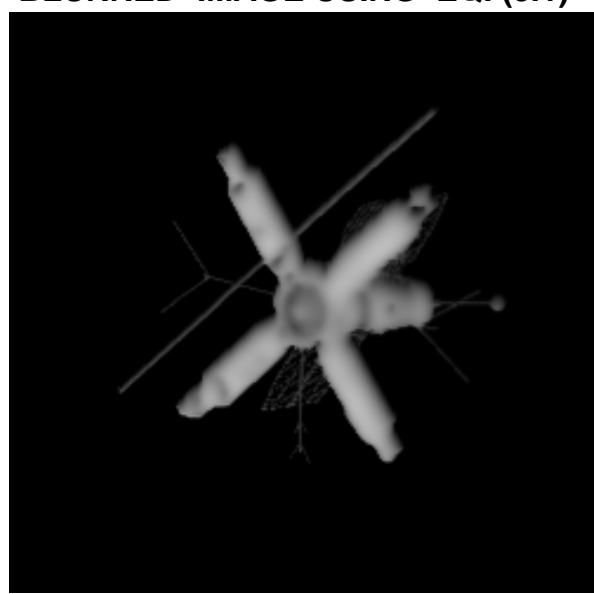

AFTER 20 VAN CITTERT ITNS.

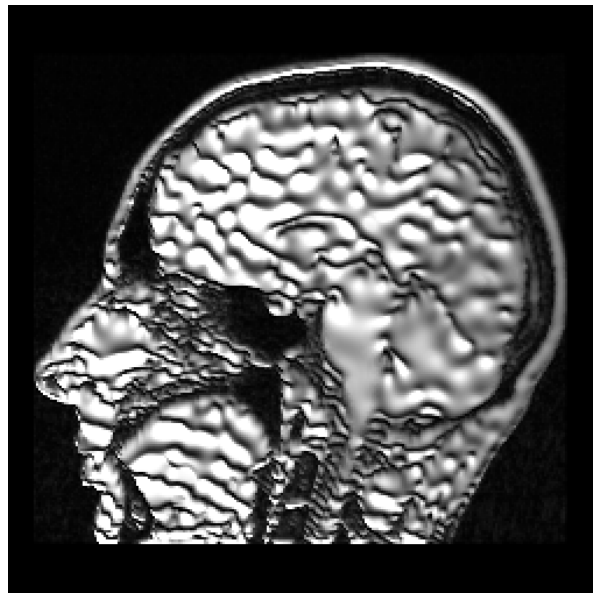

AFTER 20 VAN CITTERT ITNS.

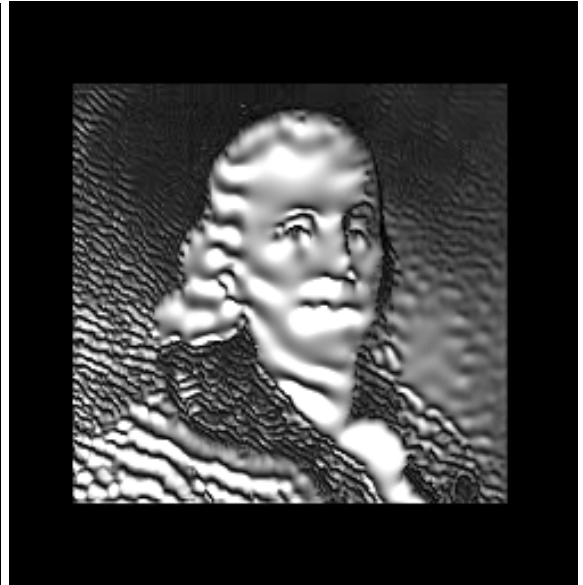

AFTER 100 VAN CITTERT ITNS.

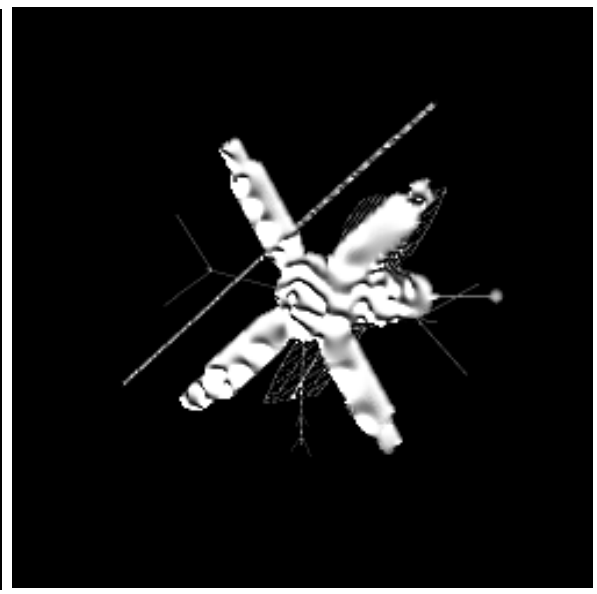

FIG. 8.2. Failed image recovery in Eqs.(3.1), (3.2), may indicate adverse Hölder-continuous behavior in stability estimate Eq. (2.2) associated with Eqs. (3.1), (3.2), and highlights possibility of untrustworthy backward continuation in other intended applications of Eqs. (3.1), (3.2). 


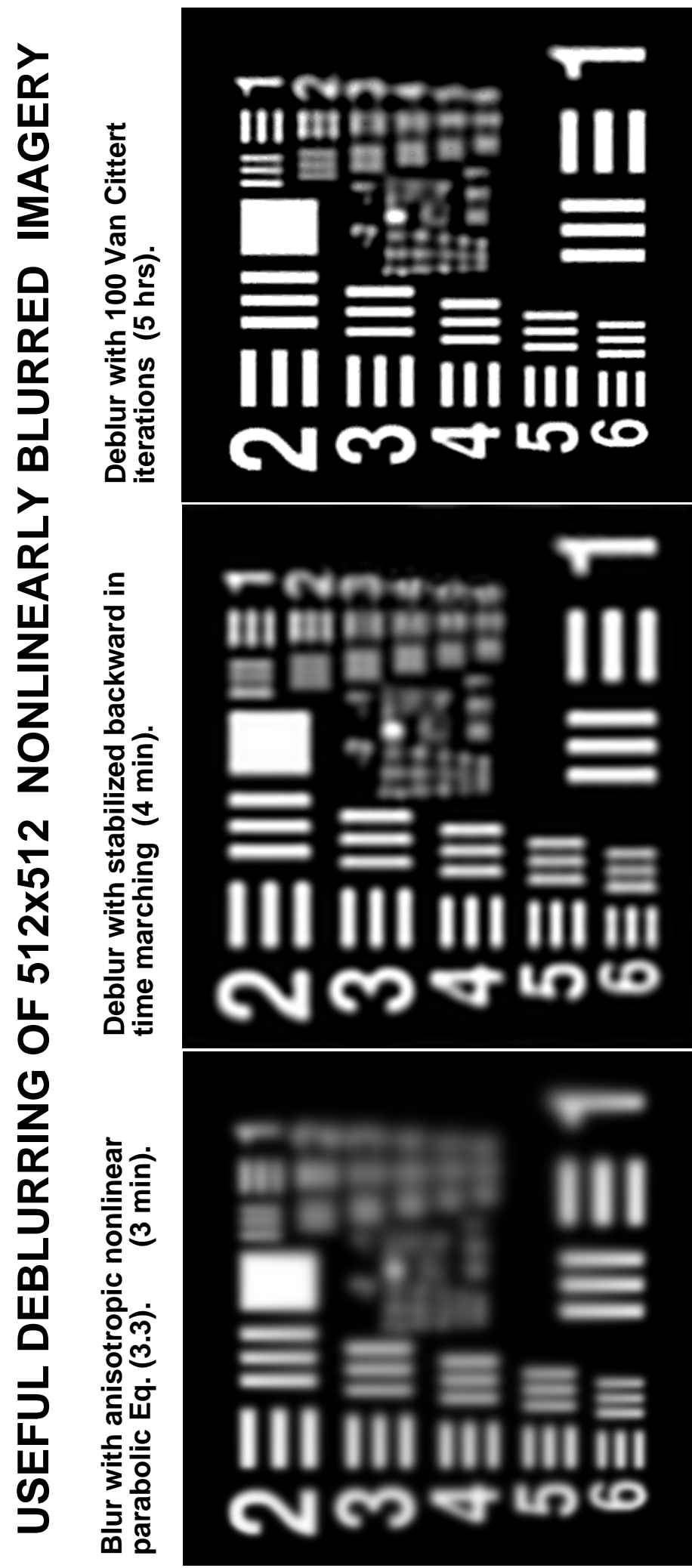




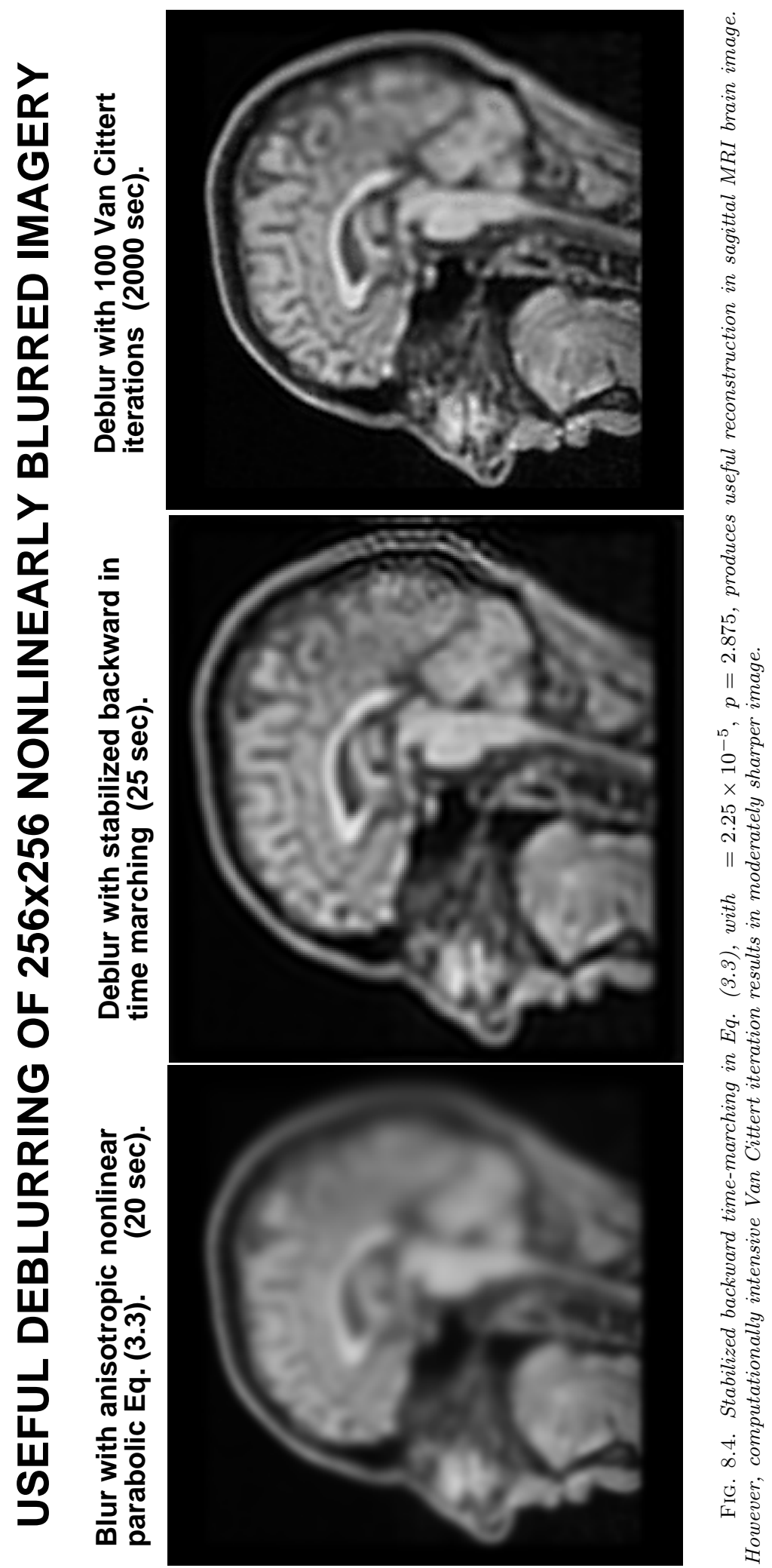




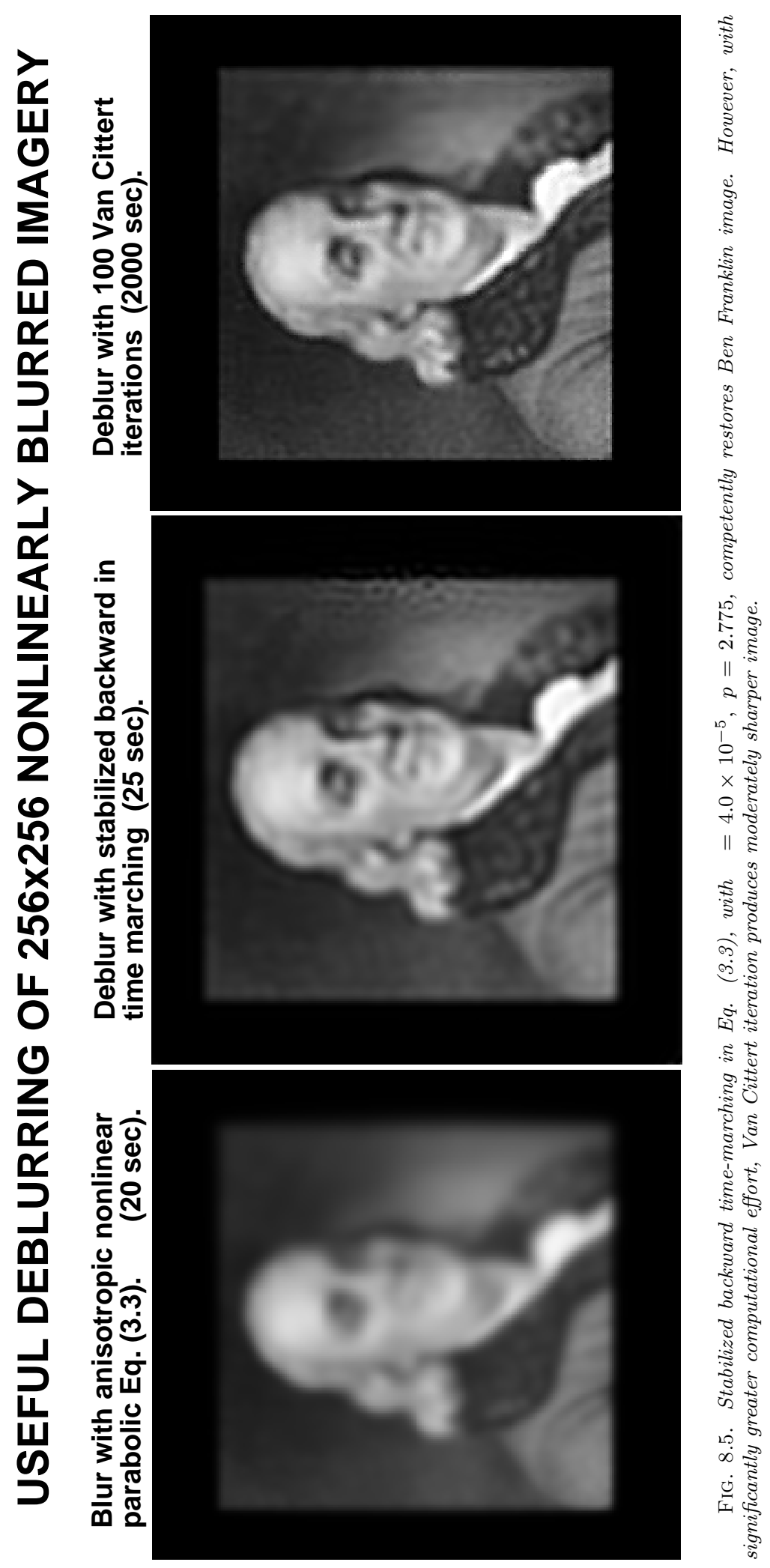




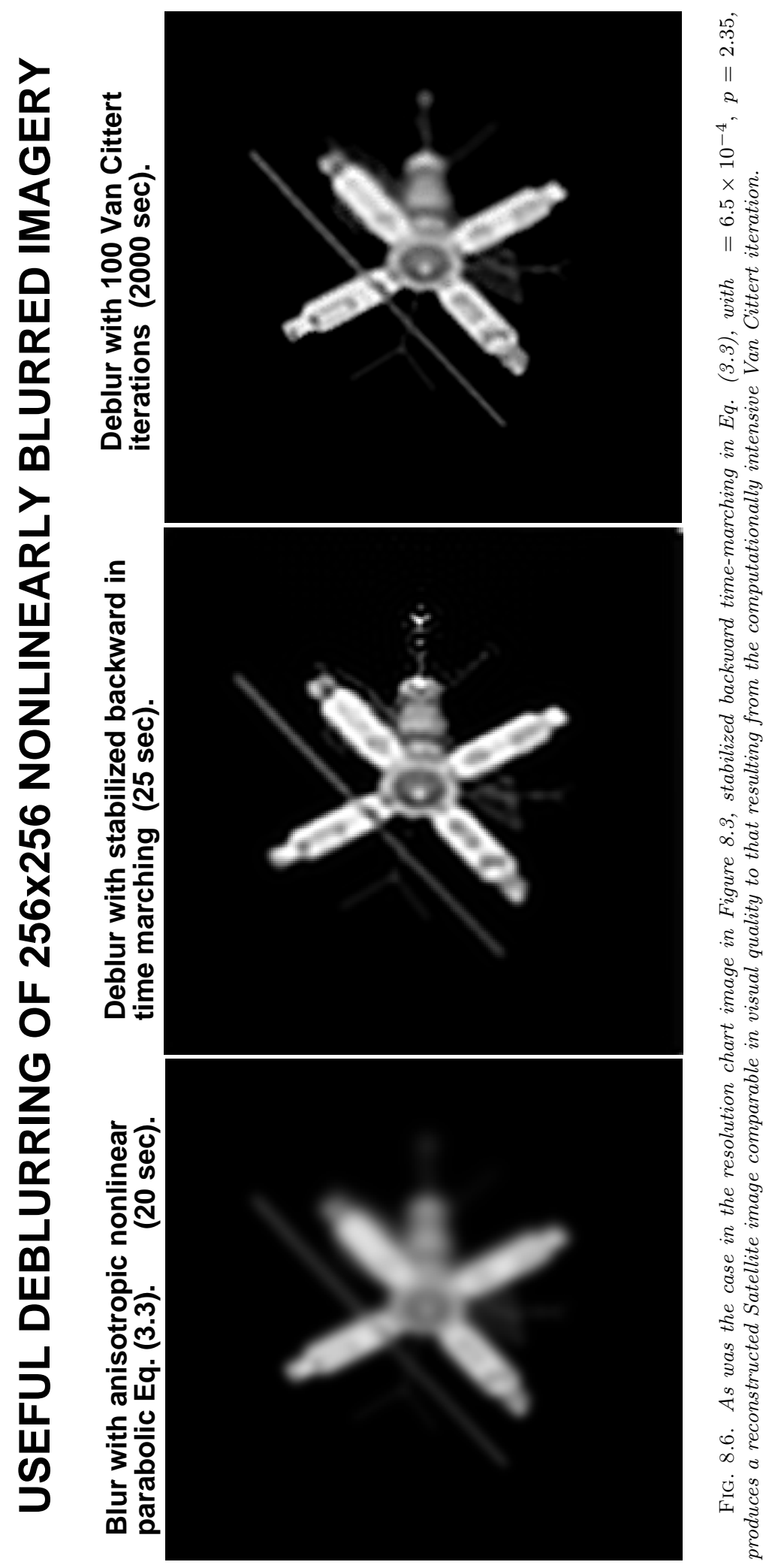


operator $-\Delta$ on $\Omega$, with the same homogeneous boundary conditions as in the original parabolic equation, precompute and store a reasonably large number of eigenvalues $\left\{\sigma_{m}\right\}_{m=1}^{M}$, with corresponding orthonormal eigenfunctions $\phi_{m}(x, y)$, where $0 \leq \sigma_{1} \leq \sigma_{2} \cdots \leq \sigma_{M}$. Analogously to the situation in Eq. (6.5), given $\epsilon, p$, let $\Delta t$ be the fixed time step in a step by step finite difference marching computation on $\Omega$. Let $v(x, y)$ be the resulting numerical solution at some discrete time $t_{k}=k \Delta t$ in the evolving computation. Expanding in the precomputed eigenfunctions of $-\Delta$, we may then define the compensating linear operator $B(\epsilon, p, \Delta t)$ applied to $v(x, y)$ as follows

$$
B v(x, y)=\sum_{m=1}^{M} \exp \left\{-\epsilon \Delta t\left(\sigma_{m}\right)^{p}\right\}\left(\int_{\Omega} v(x, y) \phi_{m}(x, y) d x d y\right) \phi_{m}(x, y) .
$$

As in Eq. (7.1), this may be incorporated in a backward time-marching algorithm on the spatial domain $\Omega$. By using $(-\Delta)^{p}$ with $p>1$, and choosing $p$ interactively, it may be possible to successfully march backward in time in difficult nonlinear parabolic equations where an adjoint spatial differential operator is ill-defined, and for which the quasi-reversibility formulation in Eq. (7.3) is inapplicable.

10. Concluding remarks. Due to the lack of reliable computational tools, backward in time continuation in multidimensional nonlinear parabolic equations remains largely uncharted territory. Two approaches were presented in this paper and applied to interesting nonlinear examples in 2D rectangular regions. The first method, introduced in [8], is a global in time nonlinear Van Cittert iterative procedure. While the linear convergence analysis is inapplicable in the nonlinear case, the Van Cittert iteration can produce useful reconstructions in a significant class of parabolic equations. However, the computational cost becomes prohibitive for large problems.

The second method is based on stepwise time-marching schemes, the universal computational approach in well-posed nonlinear evolution equations. Unfortunately, when the evolution equation is ill-posed, such schemes are necessarily computationally unstable and lead to explosive noise amplification. Here, an easily synthesized compensating operator, based on $(-\Delta)^{p}$ with $p>1$, is applied at each time step to quench the instability. In $2 \mathrm{D}$ rectangular regions, such compensating operators can be simulated using FFT algorithms. In more general domains $\Omega$, compensating operators can be simulated using a sufficiently large number of precomputed eigenpairs $\left(\sigma_{m}, \phi_{m}\right)$ for $-\Delta$ on $\Omega$, with the same homogeneous boundary conditions on $\partial \Omega$ as in the original nonlinear parabolic equation. An easily implemented compensated explicit scheme, marching backward in time, was constructed in Section 7 and applied in Section 8.

A useful idea in exploring possible backward in time continuation in a given nonlinear parabolic equation, is to associate a fictitious image deblurring problem with that equation. Numerically blurring a sharp image by marching forward in time up to some fixed time $T>0$, creates interesting noisy data that can be used to attempt reconstruction of the sharp image by backward continuation to $t=0$. Such preliminary exploration may be instructive even if the intended engineering application is unrelated to imaging. The failed reconstructions in Figure 8.2 are useful examples that signal possible untrustworthy backward continuation in Eqs.(3.1), (3.2).

The experiments in Figures 8.3 through 8.6 indicate that the compensated backward time-marching explicit scheme in Section 7 can produce reconstructions that are often comparable in visual quality to those obtained using the Van Cittert method, 
but at greatly reduced computational cost. A major advantage of the scheme in Section 7 is the ability to explore various non integer trial values of $p$ within the same program. Such versatility is not present in the quasi-reversibility method [14], and, in fact, the QR formulation in Eq. (7.3) is not readily applicable to nonlinear problems. Significantly, in the above nonlinear reconstruction experiments, simple compensating operators based on $(-\Delta)^{p}$ with $2<p<3$, were found adequate in controlling instability.

\section{REFERENCES}

[1] J. Atmadja And A. C. Bagtzoglou, Pollution source identification in heterogeneous porous media, Water Resources Research, 37 (2001), pp. 2113-2125. DOI 10.1029/2001WR000223

[2] A. C. Bagtzoglou And J. Atmadja, Mathematical methods for hydrologic inversion: The case of pollution source identification, Handb Environ Chem Vol. 5, Part F, Vol. 3 (2005): pp. 6596. DOI $10.1007 / \mathrm{b} 11442$.

[3] A. S. CARASSO, Reconstructing the past from imperfect knowledge of the present: Effective non-uniqueness in solving parabolic equations backward in time, Mathematical Methods in the Applied Sciences, (2012), DOI: 10.1002/mma.2582

[4] W. Freeden And H. Nutz, Satellite gravity radiometry as tensorial inverse problem, Int J Geomath (2011), pp. 177-218. DOI 10.1007/s13137-011-0026-x.

[5] W. Freeden, Z. NAShed, And T. Sonar (editors), Handbook of Geomathematics, 2nd edition, Springer, Heidelberg, 2013.

[6] R. J. Blakely, Potential Theory in Gravity and Magnetic Applications, Cambridge University Press, Newv York, 1995.

[7] A. S. CARAsso, Bochner subordination, logarithmic diffusion equations, and blind deconvolution of Hubble space telescope imagery and other scientific data, SIAM J. Imaging Sciences, 3 (2010), pp. 954-980.

[8] A. S. CARAsso, Hazardous continuation backward in time in nonlinear parabolic equations, and an experiment in deblurring nonlinearly blurred imagery, J. Res. NIST, 118 (2013), http:/dx.doi.org/10.6028/jres.118.010.

[9] P. H. VAN CitTert, Zum Einfluss der Spaltbreite auf die Intensittsverteilung in Spektrallinien II, Z. Physik, 69 (1931), pp. 298-308.

[10] R. J. KNops, Logarithmic convexity and other techniques applied to problems in continuum mechanics, in Symposium on Non-Well-Posed Problems and Logarithmic Convexity, R. J. Knops, ed., Lecture Notes in Math. 316, Springer-Verlag, New York, 1973.

[11] P. A. Jansson, Deconvolution with Application in Spectroscopy, Academic Press, New York, 1984.

[12] R. L. Lagendijk And J. Biemond, Iterative Identification and Restoration of Images, Kluwer Academic Publishers, Boston, 1991.

[13] A. S. Carasso, A stable marching scheme for an ill-posed initial value problem, in Improperly Posed Problems and their Numerical Treatment, (Oberwolfach Conference, September 1982). G. Hämmerlin and K. H. Hoffman, eds. Birkhäuser-Verlag, Boston, 1983.

[14] R. Lattès And J. L. Lions, The Method of Quasi-Reversibility, American Elsevier, New York, 1969. 